\section{Prolonged Weightlessness}

SIR,-It is not really too difficult to envisage the reason for prolonged weightlessness (4 July, p. 4) causing demineralization of the skeleton if Wolff's law is remembered. Not only is exercise of any significant amount presumably difficult in a space capsule, but its extent must be very limited by the need for other activities, by the postural changes that it involves if carried out with any vigour, and by the sheer boredom of repetitive, apparently purposeless, exercises.

But, in any case, deliberate exercise can cause only a fraction of the muscle-induced stresses on bone that are constantly imposed by gravity, even when changing position during sleep. As a simple example, the lumbar erector spinae have to put in a pull of at least three times the body-weight in the limited manoeuvre of bending over a basin to wash one's hands, with corresponding stresses and strains on the vertebrae. Such loads are constantly being applied to almost every bone in the body and often at much greater amplitudes. No exercise in a space-craft can begin to reproduce them, and so the feed-back mechanism $^{1}$ immediately passes the message that the bone is too massive for the work it is having to do with the result that equally immediate instructions to dismantle are received.

Unless an effective artificial gravity can be achieved, the passage of time in a state of weightlessness will inevitably have this result, and there may be a time limit, which may not be very long, perhaps as little as three or four weeks, after which the spaceman's bones will all fold up under his weight or the pull of his muscles as soon as he re-enters earth's gravity. It would seem that the only alternative for prolonged space flight would be refrigeration, assuming that this turns out to be practicable.-I am, etc.,

Canterbury.

$$
\text { F. G. St. Clair Strange. }
$$

REFERENCE

Bassett, C. A. L., Scientific American, 1965, 213

\section{Super-specialization in Surgery?}

SIR,-In the history of hip surgery many forms of operative treatment have been developed. Arthrodesis, osteotomy, mould arthroplasty, and excision all still have their useful place. More recently in Britain $\mathrm{Mr}$ G. K. McKee in Norwich, Mr. J. Charnley in Wrightington, Mr. P. A. Ring in Redhill, and surgeons at the Royal National Orthopaedic Hospital in London have developed varying types of total replacement arthroplasty. There are advantages and disadvantages in all these methods, and one hopes for continuing progress along these or other lines. That the concept and development of these operative techniques is a real advance and greatly to the credit of their initiators is enthusiastically acknowledged by many surgeons and patients. But to regard this stage as the birth of a new science seems both wrong and dangerous.

It is true that with these improved methods surgical relief is available for a greater number of patients, and this has consequently been a factor in increasing the waiting lists for admission. But throughout the country there are also other causes for this increase-delay in providing new facilities, and scarcity of geriatric convalescent beds to mention but two. Dealing with these basic factors would appear to give better and more universal cost effectiveness than establishing special centres for the treatment of one condition.

The plea by Mr. John Charnley (20 June, p. 719) for the development of a limited number of special centres would be valid if there were only a limited number of surgeons with judgement to choose the correct surgical procedure and skill to carry this out and to teach it thoroughly. I do not believe this is true. The maintenance of the present several units-one isolated, the others notdeveloping ideas and improving varying techniques by different surgeons would seem to be the optimum for continued progress. The establishment of special isolated units based solely on the surgical treatment of one or even several diseases carries with it a very real danger of a narrowing viewpoint and the retention of anachronistic methods, a point also made by Mr. Ring (4 July, p. 45).-I am, etc.,

\section{J. R. AdDISON.}

Worthing, Sussex

\section{B.M.A. Presidency}

SIR,-What is happening to the independent standing of our Association? Does no one recollect our beginnings as a union of provincial practitioners? Retired and hors concours I wish to draw attention to the repeated appointments of Maryleboners to our presidency. The Department of Health in its wisdom draws heavily on the heads of the royal colleges and societies for professional advice and guidance, and these gentlemen acquire the aura of "the establishment." Our immediate past president was also president of the Royal College of Pathologists (69-70), his successor is president of the Royal Society of Medicine (70-71), then comes a recent president of the Royal College of Obstetricians and Gynaecologists, and the Council has just announced its recommendation for (72-73) the president of the Royal College of Surgeons of England-a man of the highest merit and distinction.

Are we not in danger of losing our most important image as a completely unofficial independent body? Have the provincial practitioners and specialists no place now at the top? I know the real authority is exercised by the Chairman of the Representative Body and the Chairman of Council, but does the public know?-I am, etc.,

\section{Ewell, Surrey.}

\section{GEORGE R. MCROBERT.}

\section{The Specialty of Haematology}

SIR,-With reference to your leading article entitled "The Specialty of Haematology" (27 June, p. 743), I would like to say how difficult I find it to comprehend the muddled thinking of a learned profession which has gone on in relation to this subject for so many years. It is not however a topic that can be unravelled briefly, but I would like to attempt some solution.

For purposes of clarity I would call the physician interested in this systemic specialty a physician in blood diseases, and the pathology specialist supplying a hospital laboratory service a haematologist.

In 1962 the College of Pathologists was founded primarily because pathologists found they could not find time or practical experience to assimilate the clinical features and therapeutics required for a physician membership examination, but felt the need for a qualification of equal status to these memberships by which to measure their abilities. Thus came into being the M.C.Path., now M.R.C.Path. Why now should haematologists - that is, the pathologists doing haematology-learn again clinical features and therapeutics (in detail)? There is still no time, and if time is not there to learn therapeutics for examinations time is also not there to acquire the knowledge to practise it in those situations where examination is not sought.

The Royal College of Physicians of London, by wanting clinical haematology as a justified specialty in its own right, takes up a "physician systemic specialty" that existed in the Edinburgh Royal College of Physicians for at least a quarter of a century; the need for this branch of specialist physician care therefore seems undoubted.

Is there any problem about a qualification of M.R.C.Path. for the pathology haematologist, and M.R.C.P. for the physician in blood diseases? It must be accepted that the understanding of blood diseases does not suffer if one specialist sees the patient and another reports the blood film and marrow. What does seem necessary is that members or fellows in haematology of the Royal College of Pathologists should be granted their role of equal status and consulted along with the academic physicians who have forged their physician specialty for the Royal College of Physicians of London.

Also it is important that the pathologist, in any branch, should be seen as a doctor. Oriented thus, he becomes a member of the hospital staff. His basic function is to feed data to clinical colleagues. A skill can be taught and acquired in this art exactly comparable to the art of bedside manners between clinician and patient.

Any suggestion that the regional laboratories differ from academic departments should, in my view, be strongly resisted. Just as the academic physicians and teaching hospital physician consultants see themselves as the training source for physicians to any area, so should an academic laboratory and teaching hospital pathologists be allowed to be the training source for pathologists to all areas.

Lastly, could the Royal College of Pathologists find it acceptable to use the term histologist for the morbid anatomist/ histologist at present called pathologist. It would then have separate names for members of each of its subsections, and its generic title of pathologist available to refer to all of them. In this way I hope I have delineated two career lines-the physician in blood diseases (M.R.C.P.), and the pathologist in haematology (M.R.C.Path).-I am, etc.,

\section{MARY D. SMITH}

Department of Haematology,
Stobhill General Hospital, Glasgow N.1. 